Anaesthesist 2016 65:929-939

DOI 10.1007/s00101-016-0231-y

Online publiziert: 27. Oktober 2016

(c) Der/die Autor(en) 2016. Dieser Artikel ist

eine Open-Access-Publikation.

CrossMark

\author{
S. Mathis ${ }^{1} \cdot$ O. Schlafer ${ }^{1,2} \cdot$ J. Abram ${ }^{1} \cdot$ J. Kreutziger' $\cdot$ P. Paal ${ }^{1,3} \cdot$ V. Wenzel ${ }^{1,2}$ \\ ' Univ.-Klinik für Anästhesie und Intensivmedizin, Medizinische Universität Innsbruck, Innsbruck, \\ Österreich \\ ${ }^{2}$ Klinik für Anästhesie, Intensivmedizin, Notfallmedizin und Schmerztherapie, Medizin Campus Bodensee, \\ Friedrichshafen, Deutschland \\ ${ }^{3}$ Klinik für Anästhesie, Barmherzige Brüder Krankenhaus, Salzburg, Österreich
}

\section{Anästhesie für Medizinstudierende}

\section{Kurzanleitung zur praktischen Anästhesie bei Erwachsenen mit webbasierter Videoillustrierung}

\section{Einleitung}

In den deutschsprachigen Ländern stellen Anästhesisten die zweitgrößte Berufsgruppe unter allen Ärzten im Krankenhaus dar, aber diese Relation spiegelt sich an den meisten Universitätskliniken nicht im Ausmaß der Aufgaben in der studentischen Lehre wieder [49]. Damit ist die Wahrscheinlichkeit, dass Medizinstudierende das Fach Anästhesiologie im Studium als berufliche Perspektive für sich erkennen können, niedriger als in anderen großen Fächern. Das haben auch Wissenschaftler in Saudi-Arabien [1], Nigeria [35], Indien [25], Pakistan [32] und den USA $[5,15,41]$ beobachtet. Nachwuchssorgen sind die logische Folge [19], obwohl viele gute und wichtige Gründe für die Anästhesie als berufliche Perspektive sprechen [26].

Medizinstudierende waren Entdecker bzw. Erstbeschreiber von Spermatozoen, Äther, Penicillin, Heparin, AV-Knoten, Insulin, Langerhans-Inselzellen, Gallenblasensphincter, M. Raynaud, der RollerPumpe und Visionäre der National Library of Medicine [51]. Traditionelle Ansätze bei der Ausbildung von Medizinstudierenden bestehen in der Anästhesie häufig allein aus dem Erlernen verschiedener Strategien im Airwaymanagement oder der kardiopulmonalen Reanimation an Übungsphantomen [3, 36, 43]. Zum einen ist aber die Übertragung von ma- nuellen Fertigkeiten von einem Übungsphantom zum Patienten schwierig [42], zum anderen ist die Anästhesie viel mehr als das. Sie ist eine komplexe Interaktion aus Anatomie, Physiologie, Pharmakologie, klinischer Beurteilung, Erfahrung, Wissen und manuellem Geschick [55].

Wenn Medizinstudierende sich für eine Famulatur oder das Praktische Jahr in der Anästhesie entscheiden, besteht die einmalige Chance, dem beruflichen Nachwuchs die umfangreiche Welt der Anästhesie zu zeigen. Aber diese Chance muss auch adäquat genutzt werden: Junge Talente müssen entdeckt, gefördert und gefordert werden [29]. Die Generation Y möchte begeistert werden und ist kommunikations- sowie internetaffin [46]. Anästhesiebücher mit mehreren hundert Seiten werden, unserer Erfahrung nach, von den Medizinstudierenden als begleitende Lektüre im Studium als Informationsüberflutung angesehen. Wir haben daher eine Kurzanleitung zur praktischen Anästhesie bei Erwachsenen mit webbasierter Videoillustrierung [8] für Medizinstudierende in einer Famulatur oder dem Praktischem Jahr erstellt, um praktisches Wissen zu vermitteln und Freude für die Anästhesie zu wecken.

Diese praktischen Beschreibungen können und sollen jedoch keinesfalls das komplexe pharmakologische, physiologische und anatomische Hintergrund- wissen zur erfolgreichen Durchführung einer Anästhesie ersetzen, sondern zum schnelleren Verständnis der Anästhesie für Medizinstudenten im Operationssaal (OP) bzw. als Kurzanleitung für manuelle Fertigkeiten aufgefasst werden. In dieser Anleitung wird einer von vielen Wegen $\mathrm{zu}$ einer erfolgreichen Anästhesie beschrieben, ohne dass auf lokale Leitlinien oder „standard operating procedures" (SOP) eingegangen wird. Die angegebenen Dosierungen von Medikamenten sind Richtwerte, die anhand des individuellen Patienten, der Operation und klinischen Situation angepasst werden müssen, um Über- und Unterdosierungen $\mathrm{zu}$ vermeiden.

\section{Professionalität und Kommunikation}

In einer Famulatur oder im Praktischen Jahr wird der Student vom Patienten als Mitglied des Anästhesieteams wahrgenommen und ist damit auch ein Repräsentant der Anästhesie. Um dieser professionellen Verantwortung gerecht zu werden [12], sollte ein entsprechendes Vorgespräch stattfinden, in dem die Erwartungen (z. B. gepflegtes Äußeres, pünktliches Erscheinen, ein behutsamer, respektvoller und individueller Umgang mit dem Patienten) dem Medizinstudierenden erklärt werden. Unbedachte oder negative Suggestionen wie: „Es ist gleich vorbei.“ 
bei der Anästhesieeinleitung: „Dreh mal das Gas (volatiles Anästhetikum) auf!“ oder „Zieh mal das Gift (Opioid) auf!“ sind unprofessionell und daher unbedingt zu vermeiden [16]. Der Medizinstudent sollte dazu angehalten werden, sich allen Teammitgliedern im OP mit Namen und Funktion vorzustellen. Wenn dies nicht erfolgt, stehen nach unserer Erfahrung ca. $90 \%$ der Studierenden aufgrund ihrer eigenen Unsicherheit grußund wortlos im OP, verschränken die Arme vor der Brust und signalisieren so nonverbal ungewollt Abweisung. Dementsprechend ist die initiale Zurückhaltung des etablierten OP-Teams für eine Interaktion meistens groß und die $\mathrm{Me}$ dizinstudierenden haben so ungewollt einen unglücklichen Start. In dem Vorgespräch sollte der Student dazu ermutigt werden, bei „unakademischen“ Tätigkeiten wie Betten schieben o. ä. zu helfen. Dies wird sehr geschätzt und erleichtert die Anerkennung des OP-Teams.

\section{Anamnese, Untersuchung, Aufklärung}

Vor jeder Anästhesie muss ein Anästhesist das Risiko für jeden Patienten individuell aufgrund der Vorerkrankungen und der geplanten Operation abschätzen, darüber aufklären und ggf. weiterführende Untersuchungen bzw. Maßnahmen zur Risikominimierung anordnen. Idealerweise geschieht dies am Tage der Indikationsstellung zur Operation. Erklärt werden die Regeln (u. a. keine Nahrung bis $6 \mathrm{~h}$ vor der OP, klare Flüssigkeit bis $2 \mathrm{~h}$ vor der OP) und das Vorgehen am OP-Tag (z. B. Beruhigungstablette, Anästhesieverfahren, Zeitverlauf im OP, postoperative Schmerztherapie, Besuchszeit nach dem Eingriff) [56]. Rauchen und Kaugummi kauen am OP-Tag sind keine Gründe für ein Absagen des Eingriffs [61], sollten aber möglichst unterlassen werden. Erfragt werden u. a. Größe, Gewicht, Allergien, Vorerkrankungen, Dauermedikationen, frühere Operationen und Anästhesien (z. B. ist postoperatives Erbrechen aufgetreten?), kardiopulmonale Belastbarkeit (z. B. Dyspnoe bei welcher Belastung?), Mundöffnung, Beweglichkeit der Halswirbelsäule (wichtig für Einschätzung der Intu- bationsverhältnisse) und der Zahnstatus [28]. Eine Dauermedikation mit neuen oralen Antikoagulantien nimmt zu und ist nur schwer antagonisierbar. In diesen Fällen muss die zeitliche Dringlichkeit großer Eingriffe und die Therapieindikation (z. B. Stenteinlage in Koronargefäße mit hohem Thromboserisiko beim Absetzen) gegen das erhöhte Blutungsrisiko intraoperativ abgewogen werden [11].

Je nach der Kombination aus der Schwere des Eingriffs und Belastbarkeit des Patienten können weitere Untersuchungen wie Thoraxröntgen, Spirometrie, Ergometrie sowie Echokardiographie durchgeführt werden. In der Regel hat bei diesen Fragestellungen jedes Krankenhaus Leitlinien oder SOPs, um die Balance zwischen Risikominimierung und Kosten auszutarieren. Bei einem beschwerdefreien Patienten $<40$ Jahre reichen für einen einfachen Eingriff eine genaue Anamnese und die körperliche Untersuchung ohne Labor und EKG völlig aus [24, 28]. Eine Prämedikation ca. 30 min vor Transferierung in den OP-Saal (z. B. 7,5 mg Midazolam, $1 \mathrm{mg}$ Lorazepam) beruhigt den Patienten, verringert den Verbrauch von Anästhetika und reduziert die Inzidenz von intraoperativer Wachheit [48]. Bei ausgewählten Patienten (z. B. Demenz, Schlafapnoe, fortgeschrittene COPD, Alter >70) sollte auf eine Prämedikation verzichtet werden. Bei der Aufklärung werden dem Patienten das für den jeweiligen Eingriff bestmögliche Narkoseverfahren und mögliche Alternativen inklusive den möglichen Komplikationen erläutert. Die Wünsche des Patienten sollten berücksichtigt werden. Der mündige Patient muss dem Anästhesieverfahren zustimmen und eigenhändig unterschreiben. Je dringlicher der Eingriff (z. B. dringliche Sectio caesarea), umso weniger notwendig sind eine ausführliche Aufklärung und die schriftliche Einwilligung des Patienten. In Ausnahmefällen, z. B. bei einem schweren Trauma oder einem anderen lebensbedrohlichen Zustand, kann auf die Aufklärung verzichtet werden, um die Behandlung unverzüglich $\mathrm{zu}$ beginnen [57]. Ein Patient bewertet den Anästhesisten nicht nur an der rechtsverbindlichen Aufklärung, sondern auch an seinem Eingehen auf individuelle Sorgen und Nöte [45].

\section{Monitoring}

Nach dem Begrüßen des Patienten in der OP-Schleuse werden noch vor jeglichen medizinischen Maßnahmen die Identität des Patienten, Nüchternheitsstatus, Allergien, OP-Seite und das Vorliegen vollständiger Aufklärungsunterlagen („WHO Safe Surgery Checklist“) [53] überprüft. Vor Anästhesiebeginn müssen immer Pulsoxymetrie, nichtinvasive Blutdruckmessung und EKG angelegt werden; erweitertes Monitoring wie neuromuskuläres Monitoring bei Anwendung von Muskelrelaxantien, invasive Blutdruckmessung (z. B. bei instabilem Patient, erwartetem großen Blutverlust), zentralvenöse Katheterisierung (z. B. bei schlechtem Venenstatus, erwartete Infusion von höher dosierten Katecholaminen und speziellen Medikamenten perioperativ) und Temperatursonde (z. B. bei Eingriffsdauer $>1$ h) etc. wird im Bedarfsfall noch vor Beginn der Operation durchgeführt [14]. Das Dokumentieren der Ausgangswerte der Vitalparameter vor Anästhesiebeginn (Bewusstseinsstatus, Atmung, Kreislauf, Sauerstoffsättigungswert) ist wichtig, um eine bessere Orientierung bei der postoperativen Therapie im Aufwachraum zu gewährleisten. Die Pulsoxymetrie wird durch kalte Finger (z. B. Zentralisierung des Kreislaufs bei Hypothermie oder Schockzustand) und farbigen Nagellack negativ beeinflusst; Kohlenmonoxidvergiftung und Anämie ergeben hingegen falsch hohe Werte. Die Blutdruckmanschette sollte möglichst an dem Arm ohne Gefäßzugang angelegt werden, um eine Applikation der Medikamente und der Infusion durchgängig $\mathrm{zu}$ ermöglichen. Während der OP sollte der Blutdruck mindestens alle $5 \mathrm{~min}$ gemessen werden; bei der Anästhesieeinleitung und anderen Situationen ggf. in kürzeren Intervallen, um z. B. auf Blutdruckabfälle mit $1 \mathrm{ml}$ Akrinor $^{\circledR}$ (= Theodrenalin + Cafedrin) oder $10 \mathrm{mg}$ Ephedrin schneller reagieren zu können. $90 \%$ der kardialen Ischämien ereignen sich linksventrikulär. Bei Risikopatienten empfiehlt sich daher eine 5-Kanal-EKG- 
Anaesthesist 2016 65:929-939 DOI 10.1007/s00101-016-0231-y

(c) Der/die Autor(en) 2016. Dieser Artikel ist eine Open-Access-Publikation.

S. Mathis · O. Schlafer · J. Abram · J. Kreutziger · P. Paal · V. Wenzel

\section{Anästhesie für Medizinstudierende. Kurzanleitung zur praktischen Anästhesie bei Erwachsenen mit webbasierter Videoillustrierung}

\section{Zusammenfassung}

In den deutschsprachigen Ländern stellen

Anästhesisten die zweitgrößte Berufsgruppe unter allen Ärzten im Krankenhaus dar, aber diese Relation spiegelt sich an den meisten Universitätskliniken nicht im Ausmaß der Aufgaben in der studentischen Lehre wieder. Damit ist die Wahrscheinlichkeit, dass Medizinstudierende das Fach Anästhesiologie im Studium als berufliche Perspektive für sich erkennen können, niedriger als in anderen großen Fächern. Nachwuchssorgen sind die logische Folge, obwohl viele gute und wichtige Gründe für die Anästhesie als berufliche Perspektive sprechen.

Traditionelle Ansätze bei der Ausbildung von Medizinstudierenden bestehen in der Anästhesie häufig allein aus dem Erlernen verschiedener Strategien im Airwaymanagement oder der kardiopulmonalen Reanimation an Übungsphantomen. Die Anästhesie ist eine komplexe Interaktion aus Anatomie, Physiologie, Pharmakologie, klinischer Beurteilung, Erfahrung, Wissen und manuellem
Geschick. Wenn Medizinstudierende sich für eine Famulatur oder das Praktische Jahr in der Anästhesie entscheiden, besteht die Chance dem beruflichen Nachwuchs die umfangreiche Welt der Anästhesie zu zeigen. Junge Talente müssen entdeckt, gefördert und gefordert werden.

Wir haben eine Kurzanleitung zur praktischen Anästhesie bei Erwachsenen mit webbasierter Videoillustrierung für Medizinstudierende in einer Famulatur oder dem Praktischem Jahr erstellt. Es werden die Grundzüge der Prämedikation, der Narkoseeinleitung und Narkoseführung sowie der Regionalanästhesie erklärt. Außerdem werden die wichtigsten, für die Anästhesie relevanten Medikamente diskutiert und praktische Fähigkeiten wie das Anlegen einer Venenverweilkanüle, der Maskenbeatmung und die endotracheale Intubation anhand von Kurzfilmen erklärt. Diese Informationen sind mittels QuickResponse(QR)-Code auf einem Smartphone abrufbar. Es wurde nicht auf lokale Eigenheiten und besondere Patientengruppen (z. B. Kinder, Patienten mit speziellen Vorerkrankungen), lokale Leitlinien oder "standard operating procedures" (SOP) eingegangen. Medizinstudierende können für die Anästhesie begeistert werden, wenn man ihnen nicht nur die fachlichen Regeln und Strategien erklärt, sondern auch klinische Erfolgserlebnisse durch erlerntes und angewandtes Wissen ermöglicht. Eine übersichtliche Darstellung der Anästhesie ermöglicht bereits im Vorfeld einer Famulatur oder des praktischen Jahres eine schnelle und erfolgreiche Orientierung; ob dies anschließend auch eine Rekrutierungswahrscheinlichkeit für die Anästhesie erhöht, ist derzeit nicht bekannt.

\section{Schlüsselwörter}

Anästhesie - Medizinstudierende $\cdot$ Kurzanleitung $\cdot$ Neue Medien · Modernes Lernen

\section{Anesthesia for medical students. A brief guide to practical anesthesia in adults with a web-based video illustration}

\section{Abstract}

In Germany, Austria and Switzerland, anesthesiologists are the second largest group of physicians in hospitals, but this does not correspond to the amount of anesthesiology teaching that medical students receive in medical schools. Accordingly, the chances of medical students recognizing anesthesiology as a promising personal professional career are smaller than in other disciplines with large teaching components. Subsequent difficulties to recruit anesthesiology residents are likely, although many reasons support anesthesiology as a professional career. Traditional strategies to teach medical students in anesthesiology in medical school consist of airway management or cardiopulmonary resuscitation attempts in manikins. Anesthesiology is a complex interaction consisting of anatomy, physiology, pharmacology, clinical evaluation, experience, knowledge, and manual skills. While some medical schools offer teaching in high fidelity simulators, clinical teaching in the operating room is often limited. When medical students opt for a clinical rotation in anesthesiology, there is a chance to demonstrate the fascinating world of anesthesiology, but this chance has to be utilized carefully by anesthesiologists, as young talents have to be discovered, supported, and challenged. We have put together a short guide for medical students for a clinical rotation in anesthesiology in adults in order to generate basic knowledge and interest in anesthesiology as well as a sense of achievement. Basic knowledge about premedication, induction, maintenance and strategies for anesthesia is discussed. Further, the most important anesthesia drugs are discussed and manual skills, such as intravenous cannulation, mask ventilation, intubation, and regional anesthesia are featured with QR-code based video illustrations on a smartphone or personal computer. We did not discuss possible local mannerism and special patient groups (e. g., children, special medical history), local guidelines, or standard operating procedures.

Medical students can be inspired by anesthesiology when not simply told about the tools of the trade and strategies, but instead by gaining knowledge and clinical skills that render a sense of achievement, likely during a clinical rotation into anesthesiology. A short theoretical instruction of anesthesiology enables rapid and targeted orientation even before a clinical rotation. Whether this subsequently results in a higher recruiting chance is currently unknown.

Keywords

Anesthesia - Medical students · Short review . New media Modern learning 


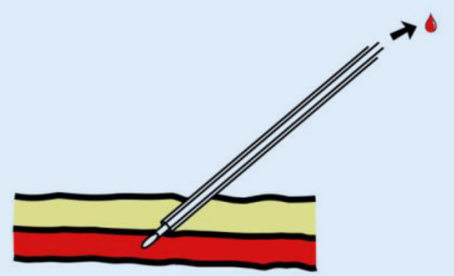

Erfolgreiche Punktion eines Blutgefäßes mit Aspiration von Blut. Achtung: Nur die Stahlkanüle liegt intravasal, nicht aber der Plastikkatheter

a

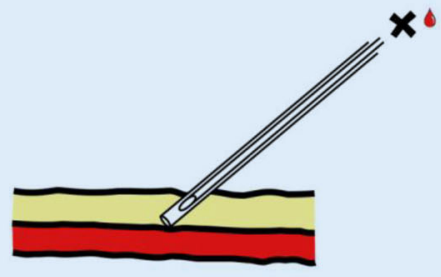

Häufiger Fehler: Die Stahlkanüle wird zurückgezogen, aber das intravasale Vorschieben des Plastikkatheter ist nicht möglich, weil er nie intravasal lag

b

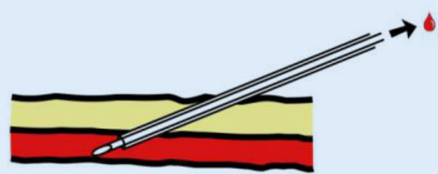

Erfolgreiche Punktion eines Blutgefäßes mit Aspiration von Blut. Danach leichtes absenken der Venenverweilkanüle. Die Stahlkanüle und der Plastikkatheter liegen intravasal

C

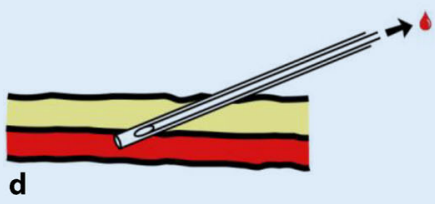

Zurückziehen der Stahlkanüle und anschließendes Vorschieben des Plastikkatheter, der dann intravasal liegt

Abb. 1 \& Platzierung einer Venenverweilkanüle. a Erfolgreiche Punktion eines Blutgefäßes mit Aspiration von Blut. Achtung: Nur die Stahlkanüle liegt intravasal, nicht aber der Plastikkatheter. b Häufiger Fehler: Die Stahlkanüle wird zurückgezogen, aber das intravasale Vorschieben des Plastikkatheters ist nicht möglich, weil er nie intravasal lag. c Erfolgreiche Punktion eines Blutgefäßes mit Aspiration von Blut. Danach leichtes absenken der Venenverweilkanüle. Die Stahlkanüle und der Plastikkatheter liegen intravasal. d Zurückziehen der Stahlkanüle und anschließendes Vorschieben des Plastikkatheters, der dann intravasal liegt

Ableitung und ein genaues Monitoring der ST-Strecke während des Eingriffs [38]. Neben dem apparativen Monitoring muss während der OP unbedingt der klinische Zustand des Patienten (Hautfarbe, Pupillengröße, Tränenflüssigkeit, Harnausscheidung) sorgfältig beobachtet und mit der Therapie abgeglichen werden, auch um das Therapieren von Messfehlern und Artefakten zu vermeiden. [58].

\section{Venenpunktion}

Außer in besonderen Situationen (z. B. Einleitung bei Kindern mit inhalativen Anästhetika) darf keine Anästhesie ohne intravenösen Zugang begonnen werden. Idealerweise sollte der Zugang soweit dis- tal wie möglich, z. B. am Handrücken, angelegt werden. Nach dem Stauen der Venen mit einem Stauschlauch sollte der Arm so tief wie möglich gelagert werden. Zum Selbstschutz sollten Einmalhandschuhe getragen werden. Nach dem Desinfizieren der Haut über der geplanten Einstichstelle sollte gewartet werden, bis das Desinfektionsmittel komplett getrocknet ist, da gewissenhaftes Desinfizieren die Infektionsrate senkt [47]. Nun wird die Vene durch leichten Druck und Zug fixiert und anschließend die Haut etwas distal der Vene im Winkel von 30-45 punktiert. Dann wird die Venenverweilkanüle waagerecht zur Haut vorgeschoben, bis Blut in die Kanüle zurückfließt. Die Stahlkanüle kann nun etwas zurückgezogen werden und der Plastikkatheter

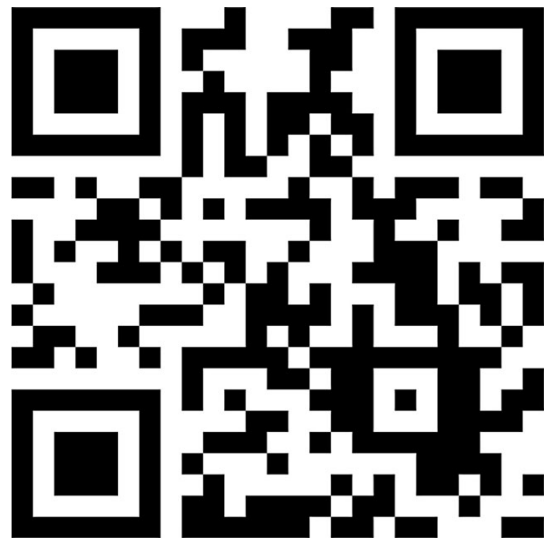

Abb. $2 \Delta$ Video 1: Venenverweilkanüle (QRCode bitte mit Smartphone einscannen). https://youtu.be/7e3VONouHAY

vorsichtig in die Vene geschoben werden. Da der Plastikkatheter nicht ganz bis zur Stahlkanülenspitze reicht, befindet sich diese beim ersten Blutstropfen noch oberhalb der Vene. Folglich ist es noch nicht möglich den Plastikkatheter intravasal vorzuschieben ( $\bullet$ Abb. 1). Die Venenverweilkanüle muss also noch ca. $1 \mathrm{~mm}$ weiter vorgeschoben werden, damit sich auch der Plastikkatheter intravasal befindet. Die Stahlkanüle kann nun etwas zurückgezogen werden und die Plastikkanüle vorsichtig in die Vene geschoben werden. Auf das Fixieren der Venenverweilkanüle (evtl. mit zusätzlicher Fixierung der Infusionsleitung am Arm) sollte besonderes Augenmerk gelegt werden, damit der Venenzugang, z. B. bei plötzlichen Bewegungen des Patienten in der Aufwachphase, nicht verloren wird (• Abb. 2).

\section{Opioide}

Opioide verhindern die Weiterleitung eines Schmerzreizes v. a. am Hinterhorn des Rückenmarks und die „Bewusstwerdung" des Schmerzes im Gehirn durch Bindung an Opioidrezeptoren; sie verhindern also nicht die Schmerzentstehung. Neben den erwünschten Wirkungen Analgesie und Sedierung kann es aber auch zu Übelkeit, Euphorie, postoperativer Hyperalgesie und Obstipation kommen. Je nach Dosis können im OP injizierte Opioide unmittelbar atemdepressiv wirken oder sogar eine Apnoe erzeugen, sodass eine engmaschige Kontrolle des Patienten unabdingbar 


\begin{tabular}{|c|c|c|c|c|}
\hline Medikament & & Initialdosis $^{\mathrm{a}}$ & Perfusordosis & Klinische Wirkdauer ${ }^{a}$ \\
\hline \multirow[t]{3}{*}{ Opioide } & Fentanyl & $2-4 \mu \mathrm{g} / \mathrm{kgKG}$ i.v. & $1-7 \mu \mathrm{g} / \mathrm{kgKG} / \mathrm{h}$ & $30-45 \mathrm{~min}$ \\
\hline & Sufentanil & $0,2-0,4 \mu \mathrm{g} / \mathrm{kgKG}$ i.v. & $0,5-1,5 \mu \mathrm{g} / \mathrm{kgKG} / \mathrm{h}$ & $30-40 \mathrm{~min}$ \\
\hline & Remifentanil & $\begin{array}{l}0,25 \mu \mathrm{g} / \mathrm{kg} / \mathrm{min} \text { bis Einset- } \\
\text { zen der Wirkung }\end{array}$ & $10-60 \mu \mathrm{g} / \mathrm{kgKG} / \mathrm{h}$ & $3-10 \mathrm{~min}$ \\
\hline Opioid-Antagonist & Naloxon & $\begin{array}{l}0,04 \mathrm{mg} \text { i.v. titrieren bis } \\
\text { zur gewünschten Wirkung }\end{array}$ & - & $60 \mathrm{~min}$ \\
\hline \multirow[t]{5}{*}{ Hypnotika } & Thiopental & $3-5 \mathrm{mg} / \mathrm{kgKG}$ i.v. & - & $10 \mathrm{~min}$ \\
\hline & Propofol & $1-2,5 \mathrm{mg} / \mathrm{kgKG}$ i.v. & $2-7 \mathrm{mg} / \mathrm{kgKG} / \mathrm{h}$ & $5-8 \mathrm{~min}$ \\
\hline & Midazolam & $0,15-0,2 \mathrm{mg} / \mathrm{KG}$ i.v. & $0,1-0,3 \mathrm{mg} / \mathrm{KG} / \mathrm{h}$ & - \\
\hline & Etomidat & $0.2-0,3 \mathrm{mg} / \mathrm{KG}$ i.v. & - & $3-4 \min$ \\
\hline & Ketamin & $1-2 \mathrm{mg} / \mathrm{kgKG}$ i.v. & $1-3 \mathrm{mg} / \mathrm{kgKG} / \mathrm{h}$ & $5-10 \mathrm{~min}$ \\
\hline $\begin{array}{l}\text { Benzodiazepin-Antagonist (z. B. für } \\
\text { Midazolam) }\end{array}$ & Flumazenil & 0,2 mg i.v.; ggf. Repetition & - & $120 \mathrm{~min}$ \\
\hline \multirow[t]{4}{*}{ Muskelrelaxantien } & Rocuronium & $0,6 \mathrm{mg} / \mathrm{kgKG}$ i.v. & - & $40 \mathrm{~min}$ \\
\hline & Mivacurium & $0,2-0,3$ mg/kgKG i. v. & - & $15-20 \mathrm{~min}$ \\
\hline & cis-Atracurium & 0,15 mg/kgKG i.v. & - & $55 \mathrm{~min}$ \\
\hline & Succinylcholin & $1-1,5 \mathrm{mg} / \mathrm{kgKG}$ i.v. & - & $7 \mathrm{~min}$ \\
\hline Antagonisierung von Rocuronium ${ }^{b}$ & Sugammadex & $4-16 \mathrm{mg} / \mathrm{kgKG}$ & - & $2-3 h$ \\
\hline $\begin{array}{l}\text { Allg. Antagonisierung von } \\
\text { Muskelrelaxanzienc }\end{array}$ & $\begin{array}{l}\text { Neostigmin } \\
\text { Robinul }\end{array}$ & $\begin{array}{l}2,0 \mathrm{mg} \text { i.v. } \\
0,4 \mathrm{mg}\end{array}$ & - & $60-90 \mathrm{~min}$ \\
\hline
\end{tabular}

ist. Für die Anästhesie kommen v. a. die Opioide Fentanyl, Sufentanil und Remifentanil zur Anwendung. Sie unterscheiden sich durch Ihre analgetische Potenz und ihre Wirkdauer voneinander. Fentanyl und Sufentanil werden meist als i. v.-Bolus zur Anästhesieeinleitung verwendet, Remifentanil wird meist mit einem Perfusor kontinuierlich infundiert, da die Halbwertszeit nur etwa 90 s beträgt (• Tab. 1). Bei der Verwendung von Remifentanil ist aufgrund der kurzen Wirkdauer auf eine ausreichende postoperative Analgesie zu achten [60].

\section{Hypnotika}

Im klinischen Gebrauch werden v. a. Thiopental, Propofol und Midazolam als Hypnotika zur Einleitung einer Allgemeinnarkose verwendet. Sie besitzen keine analgetische Komponente. Alle scheinen über eine Verstärkung der $\gamma$-Aminobuttersäure (GABA) im ZNS zu wirken. Etwa 30 s nach Injektion von Thiopental tritt eine von Atemdepression begleitete Bewusstlosigkeit ein. Nach etwa 10 min verschwindet die Wirkung von Thiopental wieder, da ein Großteil des Medikaments in Muskulatur und Fett umverteilt wird. Thiopental sollte nicht für eine kontinuierliche Infusion verwendet werden, da es aufgrund der hohen Lipophilie bei kontinuierlicher Infusion erheblich im Körperfett kumuliert. Der $\mathrm{pH}$ von Thiopental ist $\approx 10$. Paravasate müssen deshalb vermieden werden, da schwere Weichteilnekrosen entstehen können. Propofol wird in der Leber rasch abgebaut und kann deshalb auch als Dauerinfusion über einen Perfusor infundiert werden (totale intravenöse Anästhesie - TIVA). Midazolam wird häufig aufgrund seiner kreislaufinerten Wirkung zur Aufrechterhaltung der Narkose bei Intensivpatienten eingesetzt sowie zur Prämedikation verwendet. Außerdem wird es zur Sedierung, z. B. bei diagnostischen Maßnahmen, genutzt. Bei kreislaufinstabilen Patienten kann bei einer Narkoseeinleitung in Kombination mit Midazolam die Propofoloder Thiopentalgabe reduziert oder ganz eingespart werden und damit eine kreislaufstabilere Einleitung erreicht werden. Außerdem können bei kreislaufinsta- bilen Patienten Etomidat und Ketamin zur Narkoseeinleitung genutzt werden [17, 20]. Etomidat kann auch durch einmalige Injektion eine vorübergehende Hemmung der Kortisolsynthese bewirken, weshalb es in einigen Kliniken nicht verwendet wird. Ketamin wirkt über Stimulation der $\beta$-Rezeptoren kreislaufstabilisierend und scheint positive Effekte bezüglich postoperativer Schmerzen zu besitzen, weshalb es eine Renaissance im Operationssaal erfahren hat.

\section{Muskelrelaxantien}

Muskelrelaxantien werden einerseits da$\mathrm{zu}$ verwendet, die Intubationsbedingungen zu optimieren und die Stimmlippen für die Intubation zu öffnen, andererseits um intraoperativ dem Chirurgen bestmögliche Arbeitsbedingungen zu schaffen. Nichtdepolarisierende Muskelrelaxantien, z. B. Rocuronium (hepatischer Abbau), Mivacurium (renaler Abbau) oder cis-Atracurium (Abbau organunabhängig durch Plasmaesterasen) binden an der postsynaptischen 


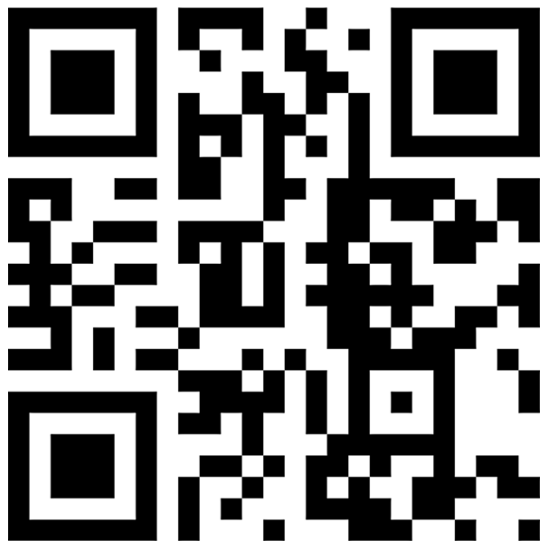

Abb. 3 ॥ Video 2: „Rapid sequence induction“ (QR-Code bitte mit Smartphone einscannen). https://youtu.be/jJGvSsiRPMM

Membran der motorischen Endplatte quergestreifter Muskulatur und verhindern so die Kontraktion des Muskels. Sie lösen aber keine Depolarisierung (= Muskelzucken) aus. Der Einsatz der Muskelrelaxantien orientiert sich nach gewünschtem Wirkungseintritt und -dauer sowie dem Abbauweg, wodurch bei unterschiedlichen Vorerkrankungen und Operationsdauern unterschiedliche Substanzen zur Anwendung kommen [31]. Wenn im Notfall eine sehr schnelle Sicherung der Atemwege notwendig ist (z. B. „rapid sequence induction“) wird häufig Succinylcholin (Anschlagszeit $60 \mathrm{~s}$, $1,0-1,5 \mathrm{mg} / \mathrm{kg}$, Wirkdauer ca. $7 \mathrm{~min}$ ) als Muskelrelaxans verwendet. Im Gegensatz zu den nichtdepolarisierenden Muskelrelaxantien bewirkt Succinylcholin eine Depolarisation der Muskelzellen, was als Muskelzucken auch makroskopisch sichtbar ist (• Abb. 3).

\section{Flüssigkeits- und Volumen- management}

Kristalloide Lösungen werden verwendet, um Flüssigkeitsverlust durch Urin, Schweiß und Atemluft zu ersetzen. Kolloidale Lösungen (gelatine- und stärkehaltige Lösungen) werden zum Ersatz von Blutverlusten und bei intravasalem Volumenmangel eingesetzt [21]. In der Regel kommen balancierte Vollelektrolytlösungen zum Einsatz, die u.a. Natrium, Kalium, Magnesium, Kalzium und zum Ausgleich der Elektroneutralität verstoffwechselbare Anionen wie
Laktat, Azetat oder Maleat enthalten. Glukose wird nur bei nachgewiesener Hypoglykämie bzw. bei Neugeborenen in geringen Dosen der Infusionslösung beigefügt. Kochsalzlösung ( $\mathrm{NaCl} \mathrm{0,9 \% )}$ sollte nicht mehr infundiert werden, da es eine hyperchlorämische Azidose verursachen kann. Anhand der „4-2-1Regel“ kann man den Flüssigkeitsbedarf pro Stunde grob abschätzen $(4 \mathrm{ml} / \mathrm{kg}$ für die ersten $10 \mathrm{~kg}, 2 \mathrm{ml} / \mathrm{h}$ für das 11.-20. $\mathrm{kg}$ und $1 \mathrm{ml} / \mathrm{kg}$ für jedes weitere $\mathrm{kg})$; also bei $70 \mathrm{~kg} \mathrm{ca} .110 \mathrm{ml} / \mathrm{h}(40+20+$ $50 \mathrm{ml} / \mathrm{h}$ ) [59], wobei der individuelle Bedarf stark von diesen Werten abweichen kann. Ein Blutdruckabfall kann mit kristalloiden Infusionslösungen aufgrund der kurzen Verweildauer im Gefäßsystem nur kurzfristig therapiert werden. Kolloidale Infusionslösungen verbleiben länger ( $>2 \mathrm{~h}$ ) im Gefäßsystem und sind so hämodynamisch besser wirksam. Während des Eingriffs ist die Infusionsstrategie den individuellen Bedürfnissen des Patienten anzupassen.

\section{Einleitung einer Allgemein- anästhesie und Masken- beatmung}

Das Anästhesiebeatmungsgerät muss vor jeder Operation vom Anästhesisten getestet werden, um Zwischenfälle durch Fehlfunktionen [18] zu vermeiden. Der Patient wird zur Anästhesieeinleitung i. d. R. waagerecht auf dem OP-Tisch in Rückenlage gelagert. Zur Operation können andere Körperpositionen notwendig sein. Während der Anästhesie ist besonders auf Druckstellen (z. B. Kabel unter dem Körper) [2] und Vermeidung von Nervenschäden zu achten (z.B. N. ulnaris am Ellenbogen, N. peronaeus lateral am Knie). Während der Operation werden die Augen mit einer Augensalbe vor dem Austrocknen geschützt und danach abgeklebt. Jeglicher Druck auf den Augapfel (z. B. bei einer Bauchlagerung) ist $\mathrm{zu}$ vermeiden, da dies eine postoperative Erblindung verursachen kann [27]. Der Patient atmet zunächst ca. 3 min $100 \%$ igen Sauerstoff über eine dicht sitzende Maske ein. Um maximale Sicherheit zu gewährleisten, sollte mit der Anästhesieeinleitung erst begonnen werden, wenn der endexspiratorische
Sauerstoff $\geq 80 \%$ beträgt [37]. Alternativ ist eine raschere Präoxygenierung mit 8 tiefen Atemzügen möglich. Nach dieser Präoxygenierung wird zur Analgesie während der Atemwegssicherung und des Eingriffs das Opioid injiziert. Dem Patienten sollte erklärt werden, dass er ein angenehmes Wärme- und Schwere-, ggf. auch Schwindelgefühl spüren wird. Wenn der Patient die Wirkung des Opioids spürt, wird das Hypnotikum injiziert, das in Kombination mit dem Opioid zu einer raschen Bewusstlosigkeit mit Apnoe führt. Ab diesem Zeitpunkt kann der Kopf vorsichtig überstreckt werden und die Maske auf Mund und Nase platziert werden. Mit dem kleinen oder Ringfinger wird der Unterkiefer am Kieferwinkel nach oben Richtung Maske gezogen und gleichzeitig die Maske mit dem „C-Griff“ durch Daumen und Zeigefinger fixiert. Am Überdruckventil des Anästhesiegeräts wird die Druckbegrenzung des Ventils auf 10-15 cm $\mathrm{H}_{2} \mathrm{O}$ eingestellt und der Patient mit dem Beatmungsbeutel beatmet. Spitzendrücke $>20 \mathrm{~cm} \mathrm{H}_{2} \mathrm{O}$ sind zu vermeiden, da dies i. d. R. zu einer Magenüberblähung führt [55]. Thoraxexkursionen und endtidales Kohlendioxid belegen eine erfolgreiche Maskenbeatmung; evtl. können Absaugen, Esmarch-Handgriff, Guedeltubus oder Halten der Maske durch eine Hilfsperson mit einem doppelten C-Griff die Beatmung verbessern [6]. Anschließend kann entweder eine supraglottische Atemwegshilfe (z. B. Larynxmaske) eingeführt werden oder es wird das Muskelrelaxans injiziert, um die endotracheale Intubation zu erleichtern (-Abb. 4).

\section{Endotracheale Intubation}

Um die Einsicht von der Mundöffnung auf den Larynx für eine erfolgreiche Intubation zu optimieren, wird der Kopf des Patienten in der „Schnüffelposition“ gelagert [44]. Der Mund des Patienten kann nun, z. B. durch Überstrecken des Kopfes und Anwendung des Kreuzgriffes bzw. leichtem Zug an den rechten oberen Zähnen, mit dem rechten Mittel-oder Zeigefinger geöffnet werden. Das Laryngoskop wird mit der linken Hand (den Griff mittig oder tief bis zum Spatel hin 


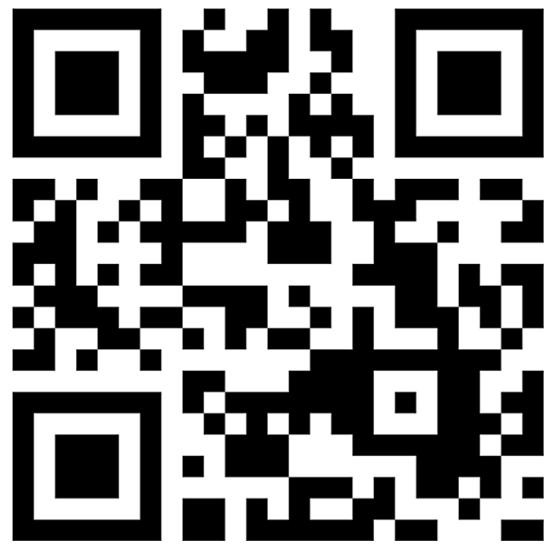

Abb. 4 \ Video 3: Maskenbeatmung (QRCode bitte mit Smartphone einscannen). https://youtu.be/DpNNOHG06WA

anfassen) vom rechten Mundwinkel unter Wegdrängen der Zunge zur Mitte des Mundes hin eingeführt. Rechts vom Laryngoskop sollte keine Zunge mehr sichtbar sein. Das Laryngoskop wird in den Winkel zwischen Epiglottis und Zungengrund vorgeschoben, bis sich die Epiglottis hebt. Es ist wichtig, den Laryngoskopgriff nur nach oben und vorne zu ziehen, ohne nach hinten über die Schneidezähne zu hebeln (•Abb.5), was schnell in Zahnschäden resultiert [33]. Der Blick auf die Glottis ist nun frei und der Tubus kann mit der rechten Hand unter Sicht durch die Stimmlippen geschoben werden. Anschließend füllt die Hilfsperson den Cuff des Tubus mit Luft (Cuffdruck ca. 25-30 mbar) und fixiert den Tubus mit Pflaster. Die Tiefe der Fixierung des Endotrachealtubus muss kontrolliert und dokumentiert werden (z. B. 19-22 cm Zahnreihe bei einem normalgroßen Erwachsenen). Neben der beobachteten, korrekten Tubusplatzierung zwischen den Stimmlippen gilt die Kapnometrie mit regulären endtidalen $\mathrm{CO}_{2}-$ Kurven am Monitor als sicheres Zeichen der endotrachealen Tubuslage. Unsichere Zeichen für eine endotracheale Intubation sind Auskultation, Thoraxexkursionen und wenn sich die Tubusinnenseite in der Ausatmung beschlägt [52]. Nach dem Präoxygenieren kann die Sauerstoffsättigung auch bei falscher Tubuslage noch einige Minuten normale Werte anzeigen. Nach einer endotrachealen Intubation sollte der Thorax auf seitengleiche Atemgeräusche auskultiert werden,

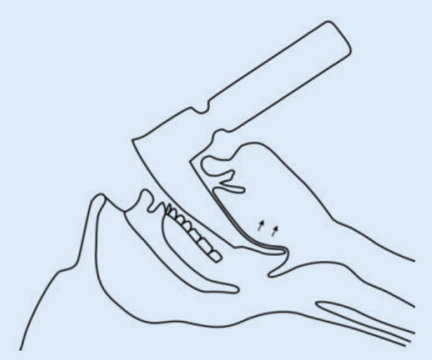

Häufiger Fehler bei der Laryngoskopie: Der Zungengrund wird angehoben, aber die Epiglottis verdeckt den Eingang der Trachea

a

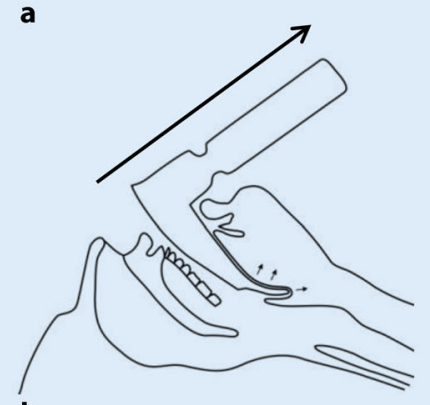

b

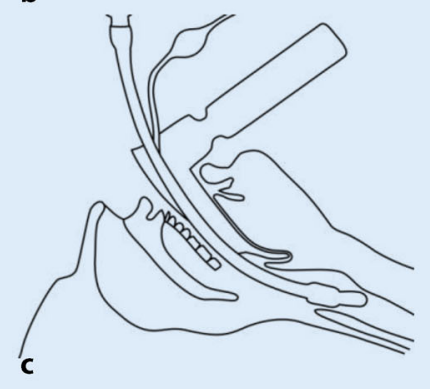

Durch vorsichtiges Positionieren des Laryngoskops in den Recessus zwischen Epiglottis und Zungengrund wird die Epiglottis angehoben, mit Zug nach oben und nach caudal/vorne, wodurch ein freier Blick auf die Trachea ermöglicht wird

Erfolgreiche endotracheale Intubation. Durch die leichte Krümmung stößt der Tubus teilweise gegen die Rückwand der Trachea; durch leichtes Drehen des Tubus und Herausziehen des Führungsstabs kann das weitere Vorschieben dann erleichtert werden

Abb. 5 A Laryngoskopie mit Einstellen der Epiglottis und endotrachealer Intubation. a Häufiger Fehler bei der Laryngoskopie: Der Zungengrund wird angehoben, aber die Epiglottis verdeckt den Eingang der Trachea. $\mathbf{b}$ Durch vorsichtiges Positionieren des Laryngoskops in den Recessus zwischen Epiglottis und Zungengrund wird die Epiglottis angehoben, mit Zug nach oben und nach caudal/vorne, wodurch ein freier Blick auf die Trachea er-möglicht wird. c Erfolgreiche endotracheale Intubation. Durch die leichte Krümmung stößt der Tubus teilweise gegen die Rückwand der Trachea. Durch leichtes Drehen des Tubus und Herausziehen des Führungsstabs kann das weitere Vorschieben dann erleichtert werden

z. B. beidseitig in der vorderen oder mittleren Axillarlinie (• Abb. 6).

\section{Anästhesiegerät, Beatmungs- formen}

Während der Allgemeinanästhesie wird der Patient meist kontrolliert beatmet, d.h. die Atmung wird vollständig vom Beatmungsgerät übernommen. Wird die volumenkontrollierte Beatmung eingesetzt, muss zunächst das Tidalvolumen (ca. 6-8 $\mathrm{ml} / \mathrm{kg}$ des Idealgewichts des Patienten) eingestellt werden, das bei jedem künstlichen Atemzug in die Lunge des Patienten gepumpt wird. Es sollte immer ein maximaler Beatmungsdruck festgelegt werden, mit dem das Tidal- volumen appliziert wird. Dieser muss ggf. adaptiert werden, weil sich die intraabdominalen bzw. intrathorakalen Druckverhältnisse, z. B. bei laparoskopischen Eingriffen oder Reanimationen, rasch verändern können [54]. Danach erfolgt das Bestimmen der Atemfrequenz pro Minute (ca. 10-14/min). Multipliziert man die Atemfrequenz mit dem Tidalvolumen, erhält man das Atemminutenvolumen (ca. 4-8 L/min). Als Frischgasfluss bezeichnet man die Menge an Gasgemisch (volatiles Anästhetikum/Sauerstoff/Luft oder Sauerstoff/ Lachgas), die pro Minute dem Kreissystem der Beatmungsmaschine zugeführt wird. Bei der Anästhesieeinleitung sollte der Frischgasfluss höchstmöglich (z. B. 


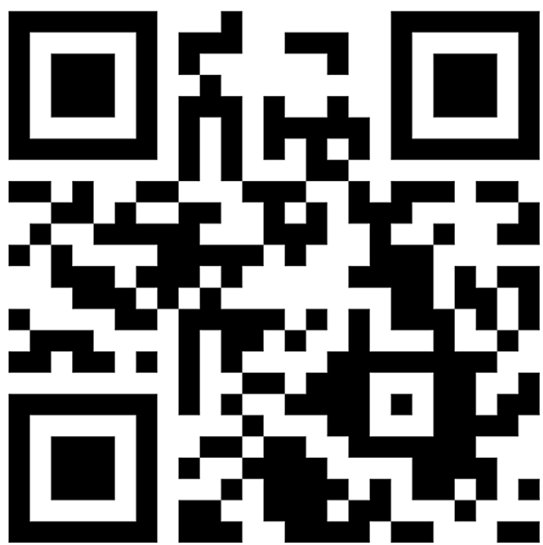

Abb. 6 ム Video 4: Endotracheale Intubation (QR-Code bitte mit Smartphone einscannen). https://youtu.be/V99Dj04Ip2c

10-18 L/min) eingestellt werden, um durch einen raschen Gasaustausch keine Vermischung der Ein- und Ausatemluft und ein rasches Anfluten von Sauerstoff zu gewährleisten. Bei der Ausleitung muss auf einen ausreichend hohen Sauerstoffgehalt geachtet werden und die Frischgaszufuhr sollte das Atemminutenvolumen übersteigen. Während des Eingriffs sollte der Frischgasfluss möglichst niedrig (z. B. 0,5-1,0 L/min) eingestellt werden, um den Verbrauch volatiler Anästhetika zu verringern und die Auskühlung über den Atemweg zu vermindern. Ein niedrigerer Frischgasfluss ist möglich, erfordert aber eine genaue Messung der inspiratorischen und exspiratorischen Sauerstoffkonzentration sowie eine hohe Vigilanz des Anästhesisten. Das Verhältnis der Inspirationszu Exspirationsdauer liegt physiologisch bei etwa 1:2 und sollte ähnlich eingestellt sein. Patienten mit obstruktiven Lungenerkrankungen haben ein verlängertes Exspirium und profitieren deswegen von einer Verlängerung der Exspirationsdauer. Der positive endexspiratorische Druck (PEEP) bezeichnet die Höhe des Drucks, der nach der Exspiration noch in der Lunge verbleibt und so hilft, die kleinen Atemwege offen zu halten. Bei Lungengesunden, normalgewichtigen Patienten wird der PEEP von etwa 5 mbar gewählt. Bei erhöhtem intraabdominellem Druck (z. B. Adipositas oder Schwangerschaft, Bauchoperationen) oder Lungenerkrankungen (z. B. Pneumonie, ARDS) kann i. d. R. mit einer Erhöhung des PEEP die Oxygenierung verbessert werden [4].

Bei der druckkontrollierten Beatmung wird der maximale Beatmungsdruck am Anästhesiegerät eingestellt, der pro künstlichem Atemzug das eingestellte Gasgemisch in die Lunge appliziert. Das dabei erreichte Tidalvolumen hängt von der Compliance der Lunge bzw. den transthorakalen Druckdifferenzen ab. Bei sich ändernden Operationsbedingungen (z.B. mit und ohne Pneumoperitoneum) muss daher auf das Atemminutenvolumen und das endtidale bzw. arterielle $\mathrm{CO}_{2}$ geachtet werden. Die restlichen Beatmungseinstellungen können analog zur volumenkontrollierten Beatmung vorgenommen werden. Bei lungengesunden Patienten wird die optimale Beatmungsstrategie kontrovers diskutiert [7]. Lungenkranke Patienten (Kontusionen, obstruktive Erkrankungen, Pneumonien, ARDS) profitieren meist von der druckkontrollierten Beatmung.

\section{Volatile Anästhetika}

Volatile Anästhetika sind leicht verdampfende Flüssigkeiten, deren Gase über die Lunge und den Kreislauf in den Körper aufgenommen werden und im ZNS hypnotisch, analgetisch und muskelrelaxierend wirken. Der Wirkmechanismus ist trotz jahrzehntelanger Anwendung im Detail noch unklar, man weiß aber mittlerweile von spezifischen Protein-, Rezeptor- und Ionenkanalwirkungen ähnlich der intravenösen Hypnotika [30]. Wie schnell ein volatiles Anästhetikum seine Wirkung entfaltet („anflutet“) hängt einerseits von der inspiratorischen Konzentration und der alveolären Ventilation ab, andererseits von der Löslichkeit des Anästhetikums im Blut sowie dem Herzminutenvolumen. Die An- bzw. Abflutungsphase verläuft umso langsamer, je größer die Löslichkeit im Blut ist. Die anästhetische Potenz eines volatilen Anästhetikums wird über die minimale alveoläre Konzentration (MAC-Wert) ausgedrückt. Ein MAC-Wert eines volatilen Anästhetikums von 1 beschreibt jene Konzentration des Volatilen, bei der $50 \%$ der Patienten nicht mehr auf einen Hautschnitt mit einem Fluchtre- flex reagieren. Je niedriger der MACWert des volatilen Anästhetikums ist, desto höher ist die anästhetische Potenz. Neben einer Reihe physiologischer Faktoren hat v. a. das Alter einen relevanten Einfluss auf die MAC: Kleine Kinder (nicht Neugeborene) haben die höchsten MAC-Werte und je älter Patienten sind, desto niedriger wird deren MAC. Derzeit stehen die volatilen Anästhetika „Isofluran“, „Sevofluran“ und „Desfluran“ in Verwendung. Sie verfügen über unterschiedliche An- bzw. Abflutgeschwindigkeit und eine unterschiedliche anästhetische Potenz. Sevofluran hat als einziges volatiles Anästhetikum einen akzeptablen Geruch und reizt die Atemwege am wenigsten, weshalb es auch zur inhalativen Einleitung (besonders relevant bei Kindern) verwendet werden kann.

\section{Spinalanästhesie}

Die Spinalanästhesie dient dazu, das Schmerzempfinden und die Muskelaktivität der unteren Körperhälfte auszuschalten. Es können also z. B. Unterbaucheingriffe (Leistenhernien, genitale Eingriffe, Sectio caesarea etc.) und Eingriffe an den Beinen (z. B. Hüfte, Knie) durchgeführt werden. Bei der Spinalanästhesie wird ein Lokalanästhetikum in den Liquorraum injiziert, wo es die Nervenwurzeln blockiert. Zum Aufsuchen der Punktionsstelle kann als Orientierung die Verbindungslinie der Beckenkämme (Spina iliaca posterior), die den Processus spinosus des vierten Lendenwirbels schneidet, herangezogen werden [9]. Beim klassischen Zugang wird median zwischen den Dornfortsätzen von L3/L4 oder L4/L5 punktiert. Ein kranial von L2/L3 gelegener Punktionsort kann zu einer Verletzung des Rückenmarks führen und ist deshalb nicht zu empfehlen. Wichtig ist eine optimale Patientenlagerung: Die Wirbelsäule sollte ohne Rotation und möglichst mit „Katzenbuckel“ (Kyphose) im Sitzen (einfacher) oder in Seitenlage gelagert werden. Die Punktionsstelle wird desinfiziert, steril abgedeckt und mit einem Lokalanästhetikum infiltriert. Danach wird eine Führungskanüle zwischen den 2 Processi spinosi sagittal vorgeschoben, anschlie- 


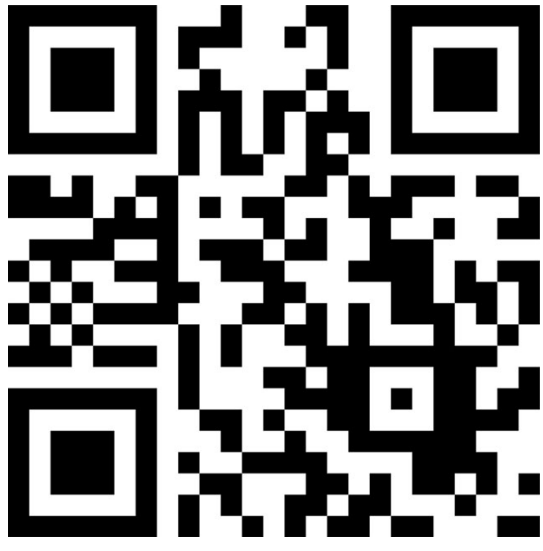

Abb. 7 \ Video 5: Spinalanästhesie (QRCode bitte mit Smartphone einscannen). https://youtu.be/bsjM22y_RjY

ßend durch die Führungskanüle mit der Spinalnadel unter Punktion der Dura mater (Duraklick) der Liquorraum erreicht. Durch Ossifikation der Ligamente oder knöcherne Anbauten kann das v. a. beim alten Patienten schwierig sein. Fließt aus der Spinalnadel klarer Liquor, kann das Lokalanästhetikum langsam injiziert werden. Die spinalanästhesiebedingte Sympathikolyse verursacht i. d. R. einen Blutdruckabfall, sodass engmaschige Blutdruck- und Herzfrequenzkontrollen sowie eine Infusion von kristalloiden und/oder kolloidalen Lösungen bzw. die Gabe von Katecholaminen erforderlich sein können (•Abb. 7).

\section{Epiduralanästhesie}

Die Epiduralanästhesie kann theoretisch auf allen Ebenen der Wirbelsäule durchgeführt werden, die zervikale Anwendung bleibt jedoch auf wenige Zentren beschränkt. Die Epiduralanästhesie kann zur intra- und postoperativen Schmerztherapie bei Eingriffen an der unteren Körperhälfte, aber auch am Oberbauch und Thorax durchgeführt werden. AuBerdem kommt die Epiduralanästhesie zur Schmerztherapie während der Entbindung zur Anwendung. Die Lagerung des Patienten und die Desinfektion der Punktionsstelle erfolgen analog zum Vorgehen bei einer Spinalanästhesie [47]. Je nach Höhe der Wirbelsäule kann ein medianer oder paramedianer Zugang gewählt werden (LWS eher median, BWS eher paramedian) und der Punktions-

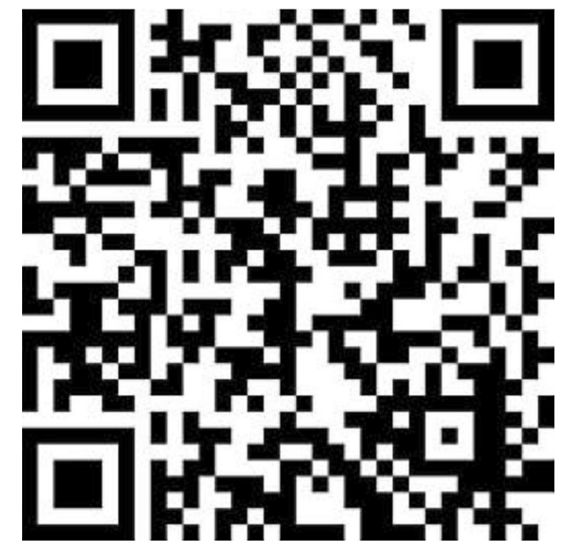

Abb. $8 \Delta$ Video 6: Periduralanästhesie (QRCode bitte mit Smartphone einscannen). https://m.youtube.com/watch?v=xtelZAnGowl\& feature=youtu.be

winkel zur Haut wird von unten nach oben immer steiler nach kranial zeigend (LWS bis BWS). Beim medianen Zugang wird nach einer Lokalanästhesie die Periduralkanüle ins Ligamentum interspinale vorgeschoben und der Mandrin der Nadel entfernt. Um den Epiduralraum zu identifizieren, wird eine spezielle leichtgängige, mit $\mathrm{NaCl} 0,9 \%$ gefüllte Spritze auf die Periduralkanüle aufgesetzt. Unter Druck auf den Stempel der Spritze wird die Kanüle nun weiter vorgeschoben. Während der Widerstand im Ligamentum flavum sehr hoch ist, verliert der Stempel beim Eindringen in den Epiduralraum rasch den Widerstand [10]. Anschließend kann durch die Kanüle ein Katheter nach kranial in den Epiduralraum geschoben werden. Um eine (spinale oder intravasale) Fehllage des Katheters auszuschließen, muss kontrolliert werden, ob Blut oder Liquor aspiriert werden kann. Wenn das nicht der Fall ist, wird zusätzlich eine Testdosis mit adrenalinhaltigem Lokalanästhetikum appliziert. Im Falle einer spinalen Lage des Katheters würde innerhalb kürzester Zeit die Wirkung einer Spinalanästhesie auftreten. Bei intravasaler Lage treten Tachykardie, EKG-Veränderungen und Blutdruckanstieg auf (•Abb. 8).

\section{Blockade peripherer Nerven}

Bei Eingriffen an den Extremitäten bietet sich eine Blockade peripherer Nerven mit oder ohne Kombination einer Allgemeinanästhesie zur intra- und postoperativen
Schmerzreduktion an. Dabei kann das Lokalanästhetikum einmalig perineural gespritzt oder ein Katheter zur kontinuierlichen Applikation eines Lokalanästhetikums eingelegt werden. Die zu blockierenden Nerven werden vorzugsweise ultraschallgezielt [13] oder alternativ mit einer Elektrostimulationsnadel aufgesucht [34]. Für Operationen an der Schulter oder am Oberarm bietet sich besonders die interskalenäre Blockade, für Operationen an Unterarm und Hand die axilläre Blockade des Plexus brachialis an. Periphere Blockaden der unteren Extremität (z. B. N. femoralis, N. ischiadicus) können ebenso mit oder ohne Allgemeinanästhesie angewandt werden und dienen ebenso der intra- und postoperativen Schmerztherapie, z. B. bei Kniegelenksersatz [40]. Eine Vielzahl weiterer peripherer Blockaden ist möglich.

\section{Postoperatives Management}

Eine suffiziente perioperative Schmerztherapie steigert den Komfort und die Zufriedenheit des Patienten, senkt die Inzidenz perioperativer Komplikationen, beschleunigt die Erholung und minimiert so auch indirekt die Kosten [39]. Der Anästhesist sollte bereits intraoperativ die Analgesie in der postoperativen Phase planen. Während des Eingriffs kann die Injektion eines Nichtopioidanalgetikums (z. B. Kurzinfusion mit 75 mg Diclofenac, 1000 mg Paracetamol oder $1000 \mathrm{mg}$ Metamizol) erfolgen. Diese Substanzen vermindern zum Teil eine Entzündungsreaktion im Gewebe und wirken so einer Schmerzentstehung entgegen. Im Aufwachraum sollte der Anästhesist ein balanciertes Schmerzmanagement aus Nichtopioidanalgetika und Opioiden (z. B. $3 \mathrm{mg}$ Piritramid oder 1-2 mg Morphium i.v.) als Bedarfsmedikation anwenden [23]. Eine Titrierung sollte erfolgen, da der individuelle Schmerzmittelbedarf sehr unterschiedlich ist. Paracetamol oder Metamizol haben bei kardiovaskulären, vaskulären, renalen oder gastrointestinalen Risiken eine günstigere Nutzen-Risiko-Relation als nichtsteroidale Antirheumatika oder Coxibe. Dagegen sind NSAR oder Coxibe nach muskuloskelettalen Eingriffen besonders wirksam, sollten aber nur 
für eine möglichst kurze Zeit und in geringst möglichen Dosierungen angewendet werden [22].

\section{Fazit für die Praxis}

Medizinstudierende können für die Anästhesie begeistert werden, wenn man ihnen nicht nur die fachlichen Regeln und Strategien erklärt, sondern auch klinische Erfolgserlebnisse durch erlerntes und angewandtes Wissen ermöglicht. Eine übersichtliche Darstellung der Anästhesie ermöglicht bereits im Vorfeld einer Famulatur oder des praktischen Jahres eine schnelle und erfolgreiche Orientierung. Ob dies anschließend auch eine Rekrutierungswahrscheinlichkeit für die Anästhesie erhöht, ist derzeit nicht bekannt.

\section{Korrespondenzadresse}

Prof. Dr. V. Wenzel, MSc., FERC

Klinik für Anästhesie, Intensivmedizin, Notfallmedizin und Schmerztherapie, Medizin Campus Bodensee

Röntgenstr. 2, 88048 Friedrichshafen,

Deutschland

v.wenzel@klinikum-fn.de

Open access funding provided by University of Innsbruck and Medical University of Innsbruck.

\section{Einhaltung ethischer Richtlinien}

Interessenkonflikt. S. Mathis, O. Schlafer, J. Abram, J. Kreutziger, P. Paal und V. Wenzel geben an, dass kein Interessenkonflikt besteht.

Für alle Videoaufnahmen liegt eine schriftliche Einverständniserklärung vor.

Open Access Dieser Artikel wird unter der Creative Commons Namensnennung 4.0 International Lizenz (http://creativecommons.org/licenses/by/4.0/deed. de) veröffentlicht, welche die Nutzung, Vervielfältigung, Bearbeitung, Verbreitung und Wiedergabe in jeglichem Medium und Format erlaubt, sofern Sie den/die ursprünglichen Autor(en) und die Quelle ordnungsgemäßnennen, einen Link zur Creative Commons Lizenz beifügen und angeben, ob Änderungen vorgenommen wurden.

\section{Literatur}

1. Abdulghani HM, Al-Shaikh G, Alhujayri AK, Alohaideb NS, Alsaeed HA, Alshohayeb IS, Alyahya MM, Alhaqwi Al, Shaik SA (2013) What determines the selection of undergraduate medical students to the specialty of their future careers? Med Teach 35(Suppl 1):25-30

2. Auerhammer J (2008) Positioning of the patient for surgery. Anaesthesist 57:1107-1124 (quiz 1125-1106)

3. Breitmeier D, Schulz Y, Wilke N, Albrecht K, Haeseler G, Panning B, Troger HD, Piepenbrock $S$ (2004) Cricothyroidotomy training on cadavers - experiences in the education of medical students, anaesthetists, and emergency physicians. Anasthesiol Intensivmed Notfallmed Schmerzther 39:94-100

4. Bruells CS, Dembinski R (2012) Positive endexpiratory pressure : adjustment in acute lung injury. Anaesthesist 61:336-343

5. Bruhn JG, Epstein BS, Burnap TK (1973) Senior medical students' knowledge of and attitudes toward anesthesiology in ten medical schools. Anesthesiology 39:94-103

6. Byhahn C, Dorges V, Graf BM (2012) Mask ventilation before relaxation. From dogma to individuality. Anaesthesist 61:397-398

7. David M, Bodenstein M, Markstaller K (2010) Protective ventilation therapy. Also relevant for the operating room? Anaesthesist 59:595-606

8. Fontelo P, Liu F, Ducut EG (2008) QR code for medical information uses. AMIA Annual Symposium proceedings, Bd. 945.

9. Gerheuser F, Crass D (2005) Spinal anaesthesia. Anaesthesist 54:1245-1267 (quiz 1268-1270)

10. Gerheuser F, Roth A (2007) Epidural anesthesia. Anaesthesist 56:499-523 (quiz 524-496)

11. Giebl A, GurtlerK(2014) New oral anticoagulants in perioperative medicine. Anaesthesist 63:347-362 (quiz363-344)

12. Goldstein EA, Maestas RR, Fryer-Edwards $K$, Wenrich MD, Oelschlager AM, Baernstein A, Kimball HR (2006) Professionalism in medical education: an institutional challenge. Acad Med 81:871-876

13. Gorsewski G, Dinse-Lambracht A, Tugtekin I, Gauss A (2012) Ultrasound-guided peripheral regional anesthesia : placement and dosage of local anesthetics. Anaesthesist 61:711-721

14. Graf J, Irqsusi M, Janssens U (2008) Hemodynamic monitoring in the perioperative period. Anasthesiol Intensivmed Notfallmed Schmerzther 43:364-372 (quiz 373)

15. Gravenstein JS, Rhoton MF (1972) Teaching anesthesia to undergraduate college students. Anesthesiology 37:641-646

16. Hansen E, Bejenke C (2010) Negative and positive suggestions in anaesthesia : Improved communication with anxious surgical patients. Anaesthesist 59:199-202

17. Haske D, Schempf B, Gaier G, Niederberger C (2014) Prehospital analgesia performed by paramedics: quality in processes and effects under medical supervision. Anaesthesist 63:209-216

18. Herff H, Paal P, Lindner KH, von Goedecke A, Keller C, Wenzel V (2008) Lachgasbedingte Todesfälle. Anaesthesist 57:1006-1010

19. Hutton P, Cooper GM (2005) Careers in anaesthesia. BrJ Anaesth 95:284-286

20. Jabre $P$, Combes $X$, Lapostolle $F$, Dhaouadi $M$, Ricard-Hibon A, Vivien B, Bertrand L, Beltramini A, Gamand P, Albizzati S, PerdrizetD, Lebail G, CholletXemard C, Maxime V, Brun-Buisson C, Lefrant JY, Bollaert PE, Megarbane B, Ricard JD, Anguel N, Vicaut E, Adnet F, Group KCS (2009) Etomidate versus ketamine for rapid sequence intubation in acutely ill patients: a multicentre randomised controlled trial. Lancet 374:293-300
21. Jacob M, Chappell D (2010) Volumenreanimation. NotfRettungsmed 13:287-293

22. Jage J, Laufenberg-Feldmann R, Heid F (2008) Drugs for postoperative analgesia: routine and new aspects. Part 1: non-opioids. Anaesthesist 57:382-390

23. Jage J, Laufenberg-Feldmann R, Heid F (2008) Drugs for postoperative analgesia: routine and new aspects: part 2: opioids, ketamine and gabapentinoids. Anaesthesist 57:491-498

24. Kratz CD, Christ M, Maisch B, Kerwat KM, Olt $C$, Zielke A, Hellinger A, Wulf H, Geldner G (2004) Premedication visits. Economizing at the cost of the patient? Anaesthesist 53:862-870

25. Kumar R, Dhaliwal U (2011) Career choices of undergraduate medical students. Natl Med J India 24:166-169

26. Landauer B (2009) Anästhesie - was denn sonst?! 10 gute Gründe Anästhesistin, Anästhesist zu werden. Anästhesiol Intensivmed 50:4-6

27. Lee LA, Roth S, Posner KL, Cheney FW, Caplan RA, Newman NJ, Domino KB (2006) The American Society of Anesthesiologists Postoperative Visual Loss Registry: analysis of 93 spine surgery cases with postoperative visual loss. Anesthesiology 105:652-659 (quiz 867-658)

28. Lingnau W, Strohmenger HU (2002) Responsibility of the anaesthesiologist in the preoperative risk evaluation. Anaesthesist 51:704-715

29. Liu EH, Lee TL (2005) The teaching of anaesthesia in Singapore. Ann Acad Med Singap 34:140C-142C

30. Loscar M, Conzen P (2004) Volatile anesthetics. Anaesthesist 53:183-198

31. Mencke T, Schmartz D, Fuchs-Buder T (2013) Neuromuscular monitoring. Anaesthesist 62:847-861

32. Minai F, UI Haq MI, Afshan G (2014) A survey of undergraduate anesthesia rotation in medical colleges of Pakistan. J Anaesthesiol Clin Pharmaco 30:82-85

33. Monaca $E$, Fock N, Doehn M, Winterhalter M, Wappler F (2010) Intubation-linked dental injuries. Relevance of individually adaptable tooth protection models. Anaesthesist 59:319-326

34. Neuburger M, Schwemmer U, Volk T, Gogarten W, Kessler P, Steinfeldt T (2014) Localization of peripheral nerves. Success and safety with electrical nerve stimulation. Anaesthesist 63:422-428

35. Nwasor EO (2010) Perception of final-year medical students about choice of anaesthesia as a specialty. Niger JMed 19:208-213

36. O'Flaherty D, Adams AP (1992) Endotracheal intubation skills of medical students. J R Soc Med 85:603-604

37. Pehbock D, Wenzel V, Voelckel W, Jonsson K, Herff $H$, Mittlbock M, Nagele P (2010) Effects of preoxygenation on desaturation time during hemorrhagic shock in pigs. Anesthesiology 113:593-599

38. Pfizenmayer H, Friederich P (2010) Perioperative monitoring of the QT interval. Anaesthesist 59:633-635

39. Reichl S, Pogatzki-Zahn E (2009) Concepts for perioperative pain therapy. A critical stocktaking. Anaesthesist 58:914-916 (918-920, 922-916 passim)

40. Reske AW, Reske AP, Meier V, Wiegel M (2009) Peripheral nerve blocks of the lower extremities. Clinical and practical aspects. Anaesthesist 58:1055-1070 (quiz 1071)

41. Rosenberg $H$ (1975) Anesthesia orientation for the medical student. Anesth Analg 54:328-331

42. Russo SG, Bollinger M, Strack M, Crozier TA, Bauer M, Heuer JF (2013) Transfer of airway skills from manikin training to patient: success of ventilation 
with facemask or LMA-Supreme ${ }^{(\mathrm{TM})}$ by medical students. Anaesthesia 68:1124-1131

43. Sakawi Y, Vetter TR (2011) Airway management and vascular access simulation during a medical student rotation. Clin Teach 8:48-51

44. Schalte G, Rex S, Henzler D (2007) Airway management. Anaesthesist 56:837-855 (quiz 856-837)

45. Schara J, Brandt L (2008) Provision of information to patients. Legal and humanitarian requirements. Anaesthesist 57:937-944 (quiz 945-936)

46. Schmidt CE, Moller J, Schmidt K, Gerbershagen MU, Wappler F, Limmroth V, Padosch SA, Bauer M (2011) Generation $Y$ : recruitment, retention and development. Anaesthesist 60:517-524

47. Schulz-Stubner S (2013) Infection prevention by the anesthesia team. Anaesthesist 62:61-76

48. Schwender D, Klasing S, Daunderer M, Madler C, Poppel E, Peter K (1995) Awareness during general anesthesia. Definition, incidence, clinical relevance, causes, avoidance and medicolegal aspects. Anaesthesist 44:743-754

49. Smith RH, Cullen SC (1963) One method of teaching anesthesia to medical students. Anesthesiology 24:68-71

50. Striebel HW (2014) Die Anästhesie. Schattauer, Stuttgart, S 109-202

51. Stringer MD, Ahmadi O (2009) Famous discoveries by medical students. ANZJ Surg 79:901-908

52. Timmermann A, Brokmann JC, Fitzka R, Nickel EA (2012) Measurement of carbon dioxide in emergency medicine. Anaesthesist 61:148-155

53. Trimmel H, Fitzka R, Kreutziger J, von Goedecke A (2013) Anesthetist's briefing check. Tool to improve patient safety in the operating room. Anaesthesist 62:53-60

54. Vogt A, Eberle B (2009) Pathophysiology of capnoperitoneum. Implications for ventilation and hemodynamics. Anaesthesist 58:520-526

55. von Goedecke A, Keller C, Voelckel WG, Dunser M, Paal P, Torgersen C, Wenzel V (2006) Mask ventilation as an exit strategy of endotracheal intubation. Anaesthesist 55:70-79

56. Weiss G, Jacob M (2008) Preoperative fasting 2008: medical behaviour between empiricism and science. Anaesthesist 57:857-872

57. Weissauer W (1999) Treatment of patients incapable of giving their consent. Legal requirements in anesthesia and intensive care medicine. Anaesthesist 48:593-601

58. Wetsch WA, Hinkelbein J, Spöhr F (2014) Kurzlehrbuch Anästhesie, Intensivmedizin, Notfallmedizin und Schmerztherapie. Thieme, Stuttgart

59. Zausig YA, Weigand MA, GrafBM (2006) Perioperative fluid management: an analysis of the present situation. Anaesthesist 55:371-390

60. Zollner C, Schafer M (2008) Opioids in anesthesia. Anaesthesist 57:729-740 (quiz 741-722)

61. Zwissler B, Reither A (2005) Preoperative abstinence from smoking. An outdated dogma in anaesthesia? Anaesthesist 54:550-559

\section{Preise der DGAI}

\section{Karl-Thomas-Preis}

Der von der Firmengruppe B. Braun Melsungen AG gestiftete Karl-Thomas-Preis in Höhe von 2.500 Euro für bedeutsame Arbeiten auf dem Gebiet der Anästhesiologie, Intensivund Notfallmedizin steht auch zur Preisvergabe 2017 zur Verfügung.

Um den Preis können sich alle Mitglieder der Deutschen Gesellschaft für Anästhesiologie und Intensivmedizin bewerben. Die näheren Teilnahmebedingungen ergeben sich aus den Statuten des Karl-Thomas-Preises (Anästh. Intensivmed. 9/1991, 276).

Gutachterkommission:

Prof. Dr. Dr. K. Zacharowski, Frankfurt (federführend)

Prof. Dr. B. Pannen, Düsseldorf

Prof. Dr. P. Rosenberger, Tübingen

Stellvertreter:

Prof. Dr. K. Engelhard, Mainz

Prof. Dr. P. Zahn, Bochum

\section{August-Bier-Preis}

Der von der Firma Pajunk Medical Produkte $\mathrm{GmbH}$, Geisingen, gestiftete August-Bier-

Preis in Höhe von 2.500 Euro für bedeutsame Arbeiten auf dem Gebiet der klinischen Regionalanästhesie steht auch zur Preisvergabe im Jahre 2017 zur Verfügung.

Um den Preis können sich alle Mitglieder der Deutschen Gesellschaft für Anästhesiologie und Intensivmedizin bewerben. Die näheren Teilnahmebedingungen ergeben sich aus den Statuten des August-Bier-Preises (Anästh. Intensivmed. 11/1997, 588 f.).

Gutachterkommission:

Prof. Dr. Th. Volk, Homburg

(federführend)

Prof. Dr. W. Gogarten, Bielefeld

Prof. Dr. F. Wappler, Köln

Stellvertreter:

Prof. Dr. D. Bremerich, Limburg

Priv.-Doz. Dr. Th. Grau, Gütersloh

\section{Carl-Ludwig-Schleich-Preis}

Der von der Firma Grünenthal $\mathrm{GmbH}$, Aachen, gestiftete Carl-Ludwig-Schleich-Preis in Höhe von insgesamt 2.500 Euro für bedeutsame Arbeiten auf dem Gebiet der Schmerztherapie steht erneut zur Preisvergabe im Jahre 2017 zur Verfügung.

Um den Preis können sich alle Mitglieder der Deutschen Gesellschaft für Anästhesiologie und Intensivmedizin bewerben. Die näheren Teilnahmebedingungen ergeben sich aus der Satzung für die Vergabe des Carl-Ludwig-Schleich-Preises (Anästh. Intensivmed. 11/2001, 912).

Gutachterkommission:

Prof. Dr. W. Koppert, Hannover

(federführend)

Prof. Dr. C. Nau, Lübeck

Prof. Dr. E.M. Pogatzki-Zahn, Münster

Stellvertreter:

Prof. Dr. S. Ch. Azad, München

Prof. Dr. W. Meissner, Jena

\section{Heinrich-Dräger-Preis für Intensivme- dizin}

Der von der Dräger Medical AG \& Co. KGaA Lübeck, gestiftete Heinrich-Dräger-Preis für Intensivmedizin in Höhe von insgesamt 2.500 Euro für herausragende Arbeiten und Projekte auf dem Gebiet der Intensivmedizin steht auch zur Preisvergabe im Jahre 2017 zur Verfügung.

Um den Preis können sich alle Mitglieder der Deutschen Gesellschaft für Anästhesiologie und Intensivmedizin bewerben. Die näheren Teilnahmebedingungen ergeben sich aus den Statuten für die Vergabe des HeinrichDräger-Preises für Intensivmedizin (Anästh. Intensivmed. 12/2004, 748).

Gutachterkommission:

Prof. Dr. G. Marx, Aachen

(federführend)

Prof. Dr. M. Gama de Abreu, Dresden

Prof. Dr. Ch. Werner, Mainz

Stellvertreter:

Priv.-Doz. Dr. J.-P. Roesner, Rostock

N.N.

Berücksichtigt werden Arbeiten, die bis zum 31. Januar 2017 (Datum des Poststempels) an die DGAI-Geschäftsstelle, Roritzerstraße 27, 90419 Nürnberg, per Einschreiben abgegangen sind. Die Arbeiten werden in dreifacher Ausfertigung erbeten; zusätzlich auch in elektronischer Fassung an: klanger@dgai-ev.de. In einem Begleitschreiben ist mitzuteilen, ob und gegebenenfalls wo die Arbeit erstmals veröffentlicht wurde. Es ist außerdem die Erklärung abzugeben, dass die Arbeit für keinen anderen Preis eingereicht worden ist. 